\title{
DILEMAS BIOÉTICOS Y SUS POSIBLES SOLUCIONES EN LAS UNIDADES DE TERAPIA INTENSIVA PEDIÁTRICA DEL DISTRITO FEDERAL (MÉXICO)
}

\author{
Ma. de la Luz Casas Martínez', Ma. Cristina Caballero Velarde²
}

Resumen: El proceso de toma de decisiones en las unidades de terapia intensiva pediátrica (UTIP) se sustenta en un marco científico, legal y ético, el cual suele ser más complejo que en la práctica para adultos e involucra a los padres o tutores, en consecuencia, debe ser resuelto con seriedad, con calma y rigor metodológico. Se diseñó una encuesta por expertos, con aplicación anónima y voluntaria, en forma no institucional, en la que se plantearon cuestiones referentes a dilemas bioéticos generados en los servicios de UTIP y los procedimientos para su solución. La aplicación de este instrumento fue el método Delphi, en dos rondas. Los resultados más importantes, entre otros, consistieron en reconocer que la totalidad de los médicos encuestados enfrentan en su unidad dilemas éticos, siendo el más frecuente la obstinación terapéutica, en un 32\%, resultado que se enlaza al de la dificultad de establecer el diagnóstico de paciente terminal, en un 13\%. Incluir materias de bioética y toma de decisiones con contenido moral en la formación de estos profesionistas, así como implementar la comunicación asertiva entre el equipo de salud y la familia, y promover el funcionamiento adecuado de comités de bioética hospitalarios con funciones consultivas de emergencia, son propuestas que emergen de las conclusiones de este estudio.

Palabras clave: terapia intensiva, pediatría, bioética

Bioethics dilemmas and their possible solutions in pediatric intensive therapy units of Federal District (Mexico)

Abstract: The process of decision making in pediatric intensive therapy units (PITU) is based on an ethical, legal and scientific framework, which generally is more complex that with adults and involves parents or tutors, thus, it must be solved carefully, with time and methodological precision. A survey was design for experts with anonymous and voluntary application, in a non institutional way, in which questions arise referring to bioethical dilemmas generated in PITU services and proceedings for their solution are proposed. Delphi method was used as instrument in two rounds. The most important results, among others, consisted in recognizing that all physician surveyed face in their unit ethical dilemmas, the most frequent being obstinacy in therapy in $32 \%$ cases, result linked to the difficulty to establish the diagnosis of terminal patient in $13 \%$ cases. To include bioethics topics and decision making with moral content in training professionals, as well as implementing assertive communication among health care team and families, and promoting adequate functioning of hospital bioethics committees with emergency consulting functions, are proposals which arise from the conclusions of this study.

Key words: intensive therapy, pediatric, bioethics

Dilemas bioéticos e suas possíveis soluçóes nas Unidades de Terapia Intensiva Pediátrica do Distrito Federal (México)

Resumo: O processo da tomada de decisôes nas Unidades de Terapia Intensiva Pediátrica (UTIP) se sustenta num marco científico, legal e ético, que deve ser mais complexo do que na prática para adultos, e envolve os pais ou tutores; em consequência, deve ser resolvido com seriedade, calma e rigor metodológico. Planejou-se uma enquete por especialistas, com aplicaçáo anônima e voluntária, de forma não institucional, na qual foram propostas questóes referentes a dilemas bioéticos gerados nos serviços de UTIP e os procedimentos para a sua solução. A aplicação deste instrumento se deu pelo método Delphi, em duas etapas. Os resultados mais importantes, entre outros, consistiram em reconhecer que a totalidade dos médicos pesquisados enfrentam em sua unidade dilemas éticos, sendo o mais frequente a obstinação terapéutica, em $32 \%$ dos casos, resultado que se relaciona ao de dificuldade em estabelecer o diagnóstico de paciente terminal, em 13\%. Incluir matérias de bioética e tomada de decisôes com conteúdo moral na formação destes profissionais, assim como implementar a comunicação assertiva entre a equipe de saúde e a família, e promover o funcionamento adequado de Comitês de Bioética hospitalares com funçóes consultivas de emergência, são propostas que emergem das conclusôes deste estudo.

Palavras-chave: terapia intensiva, pediatria, bioética

\footnotetext{
${ }^{1}$ Escuela de Medicina de la Universidad Panamericana, México Correspondencia: mcasas@up.edu.mx

${ }^{2}$ Unidad de Terapia Intensiva Pediátrica Centro Médico Nacional 20 de noviembre, México
} 


\section{Introducción}

Asclepio, según la mitología griega, fue criado y educado por el centauro Quirón, quien lo instruyó en las artes de la medicina y de la caza. La diosa Atenea, para favorecerle, le entregó dos redomas llenas de sangre de la Gorgona. En una de ellas la sangre estaba envenenada y en la otra tenía propiedades para resucitar a los muertos. El joven Asclepio, poseedor de la vida y la muerte, llegó a dominar el arte de la resurrección. Desde entonces, el ejercicio de la medicina, está ligado a este inmenso poder.

Veinticinco siglos después los médicos han realizado todo noble esfuerzo por conservar la vida de aquellos confiados a su saber. El avance de la tecnología dio origen a las unidades de terapia intensiva (UTI), servicios ligados simbólicamente a la posibilidad de la resurrección, según el mito de Asclepio, ya que en ellas pudo salvarse a aquellos antes insalvables. En 1992 queda registrada ante la División de Estudios de Postgrado de la Universidad Nacional Autónoma de México (UNAM) la subespecialidad de Medicina del Enfermo Pediátrico en Estado Crítico. En esos momentos, su contribución a la medicina pediátrica parecía solo brindar beneficios, se hacía lo inexplicable por prolongar una vida con la mejor intención; pero, sin la adecuada comprensión de los límites terapéuticos, muchas veces estos esfuerzos llenaron de incertidumbre al mundo médico y a la sociedad, situando sus acciones en el campo de la obstinación terapéutica. El hecho es que la capacitación de estos nuevos especialistas se centraba en la tecnología, pero no incluía la oportuna reflexión ética ni el diagnóstico de "paciente terminal", tampoco la directriz integral de las importantes decisiones que derivan e impactan en la evolución de la enfermedad. La reflexión propiciada por casos emblemáticos, como el de Karen Ann Quinlan, hace que, en forma lenta pero progresiva, la bioética se haga presente, dando inicio a un incansable diálogo entre la tecnología biomédica, los límites de la ciencia y los cambios sociales y culturales de la presente época.

La bioética se ha convertido ahora en un inagotable e indispensable espacio de reflexión, que analiza los conflictos morales que surgen entre el uso correcto del avance tecnológico, la práctica clínica y la investigación(1).
El proceso de la toma de decisiones en cuidados intensivos pediátricos se sustenta en un marco científico, legal y ético, que suele ser más complejo que en la práctica para adultos y que involucra a los padres o tutores; en consecuencia, debe ser resuelto con seriedad y rigor metodológico.

El binomio ciencia-reflexión ética despierta un creciente interés en el ámbito de los cuidados intensivos en general. Tal relación busca siempre apoyo en los principios de la bioética — no maleficencia y beneficencia, autonomía o respeto por la persona, y justicia distributiva(2)—, así como en el principio de proporcionalidad, que sustenta la obligación moral de implementar solo aquellas medidas terapéuticas que guarden una relación de debida proporción entre los medios empleados y el resultado previsible(3).

El objetivo del presente trabajo fue examinar mediante un estudio de encuesta por expertos entre el personal médico de UTIP de hospitales públicos del Distrito Federal- la percepción que este personal tiene de los dilemas éticos, las repercusiones, la problemática consecutiva, así como conocer las sugerencias de alternativas posibles de solución en dilemas semejantes entre las diferentes UTIP participantes.

Se trata de un trabajo inédito en nuestro país, confirmado por revisión bibliográfica por el Centro de Conocimiento Bioético de la Comisión Nacional de Bioética Mexicana (CONBIOETICA).

Los términos utilizados en éste trabajo se apegan a las definiciones del Glosario de términos y expresiones frecuentes de bioética en la práctica de cuidados intensivos pediátricos de Hernández Rastrollo R. y col.(4).

\section{Material y métodos}

Se diseñó una encuesta por expertos, con aplicación anónima y voluntaria, en forma no institucional, en la que se plantearon cuestiones referentes a dilemas bioéticos generados en los servicios de UTIP y los procedimientos para su solución. La aplicación de este instrumento fue el método Delphi, en dos rondas. Se contó con el registro de esta investigación en el Comité de Ética de la Investigación de la Escuela de Medicina de la Uni- 
versidad Panamericana, México. La investigación se clasificó como riesgo tipo I por el Reglamento de Investigación de la Ley General de Salud Mexicana.

El formulario se envió por correo electrónico a médicos especialistas en pediatría y en terapia intensiva, con ejercicio profesional de 15 ańos o más en la atención al niño gravemente enfermo, que laboran en UTIP de hospitales públicos en el DF, con certificación de posgrado por la UNAM. El universo de estas unidades es de 7 , siendo la muestra representativa del $85 \%$ de ellas. Se asignó un código a cada centro.

Fases

- Entrevista personal por correo electrónico, con base en una encuesta no estructurada con once preguntas abiertas.

- Compilación de las respuestas.

- Ronda de reflexión y análisis de los mismos participantes a través de envío de las respuestas del grupo.

- Compilación y análisis de las respuestas en segunda ronda.

- Análisis de los resultados en forma descriptiva, con expresión porcentual y de tendencia central.

- Análisis y reflexión sobre las respuestas.

\section{Encuesta}

Fase 1

1. ¿Cuales son los dilemas bioéticos mas frecuentes en la atención al niño grave?

2. ¿Cuál (es) son en su opinión las posibles soluciones?

3. ¿ La relación médico-paciente-familia se ha visto afectada?

Si No

4. ¿Ha enfrentado demandas?

Si No
5. ¿La relación interpersonal con el resto del equipo médico de trabajo se ha visto afectada?

Si

No

6. ¿Ha motivado incremento del Sindrome de Bourn out en el personal médico?

Si No

7. ¿Le motiva delegar en otro médico la responsabilidad de los casos con dilema ético?

Si No

8. ¿Existe temor del personal médico a posibles demandas juridicas?

$$
\text { Si No }
$$

9.- Al tomar una decisión en torno a dilemas (LET, ONR, negativa de ingreso a UTIP, etc.), ¿existe duda si es éticamente correcta?

Si No

10.- ¿Ha repercutido en forma personal el tomar este tipo de decisiones? (ansiedad, depresión, molestia etc.)

Si No

(especificar)

Fase 2

Una vez completada la encuesta se envió en forma anónima, a cada uno de los médicos participantes, todas las respuestas. Una vez valoradas la totalidad de las respuestas y opiniones se les solicitó dar respuesta a la siguiente pregunta:

Como segunda parte de la presente encuesta, favor de informar:

11. ¿Modifica parcial o totalmente sus respuestas o bien ratifica las mismas?

\section{Resultados}

Se recibieron 22 encuestas, contestadas por médicos de seis UTIP de hospitales que cuentan con el Programa Único de Especialidades Médicas (PUEM), avalado por la División de Estudios de Postgrado de la UNAM. 
La totalidad de los médicos encuestados reconoció que, en el ejercicio de su actividad médica asistencial, enfrenta en su unidad dilemas éticos, siendo el más frecuente la obstinación terapéutica, en un $32 \%$, resultado que se enlaza al de dificultad de establecer el diagnóstico de paciente terminal en un $13 \%$. La determinación de terminalidad en el paciente pediátrico crítico es, con frecuencia, una condición de extrema dificultad, ya que implica establecer con relativa seguridad que un paciente es irrecuperable. En este sentido, la decisión busca apoyo en el uso de indicadores cuantitativos de pronóstico de morbi-mortalidad, como el PRIMS, ITT, PELOD, entre otros $(5,6$,); sin embargo, es prudente considerar que los niños tienen una mayor reserva biológica y, en consecuencia, poseen gran capacidad de recuperación, y muchas veces su evolución es sorprendente, tanto en el pronóstico vital como en las potenciales secuelas(7).

Reconociendo la dificultad al momento de decidir sobre el internamiento de niños con enfermedades oncológicas y disfunción orgánica múltiple, en quienes el pronóstico de su recuperación es muy incierto, el 23\% de los médicos participantes respondieron afirmativamente. La valoración del alcance de la autonomía en el paciente pediátrico y en su familia se manifestó en duda en el 16\%.

Un problema ético también referido fue la falta de conocimientos en bioética, no obstante ser médicos con experiencia laboral de 15 ańos o más, se menciona en un $13 \%$. Hubo opiniones en cuanto a la dificultad de decisión en las órdenes de no reanimación en un $4 \%$, considerando que los pacientes son irrecuperables y pueden quedar con daño neurológico severo post paro (gráfico 1).

\section{Gráfico 1: Dilemas Bioéticos UTIP}

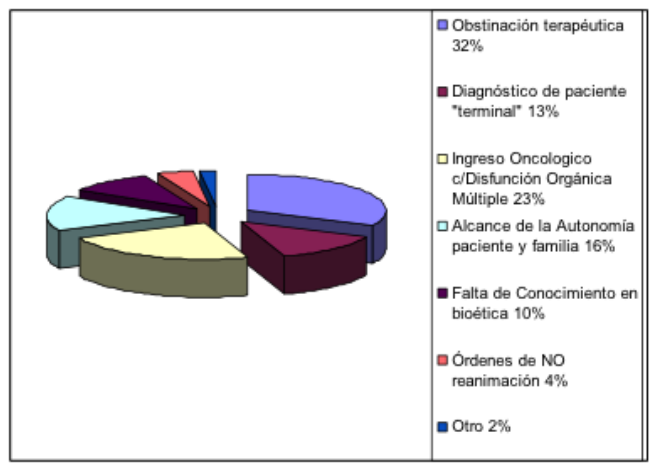

Respecto de la búsqueda de posibles soluciones, los participantes respondieron en primer lugar "adquirir conocimientos en bioética", en un $27 \%$. Sigue en porcentaje, con un $22 \%$, contar con un Comité Hospitalario de Bioética (CHB) que, al ser requerido, colabore en la toma de decisiones. Cabe considerar que los centros hospitalarios de las UTIP participantes cuentan en su totalidad con $\mathrm{CHB}$.

Obtener un acuerdo por consenso entre el personal responsable de la atención al niño; en consecuencia, la elaboración de guías de apoyo para la toma de decisiones se valoró como acción necesaria por el $16 \%$ de los participantes.

Los participantes refieren el deseo de contar con leyes adecuadas que brinden seguridad en la toma de decisiones difíciles en el niño grave con un $12 \%$. Un $10 \%$ de los encuestados señalan la importancia de involucrar a la familia en la toma de decisiones (gráfico 2).

\section{Gráfico 2: Alternativas de solución}

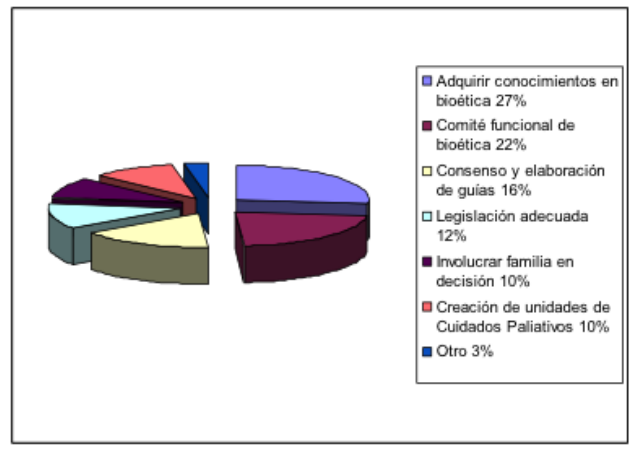

La creación de unidades de cuidados paliativos fue referida como un aspecto de solución a los dilemas bioéticos en un $10 \%$. A la pregunta de si la relación médico-paciente-familia se ha visto afectada con las decisiones tomadas en contexto UTIP, el 32,5\% respondió afirmativamente.

De los participantes en el estudio, el 37,5\% refirió haber enfrentado alguna demanda, y algunos mencionaron que fue en una sola ocasión. Cabe considerar que, acorde con la procedencia de la encuesta, se confirma que corresponden a dos de las UTIP participantes y con probabilidad se referían a los mismos casos. 
Respecto de la posibilidad de afectación de la relación interpersonal con el resto del equipo de trabajo, predominó la respuesta negativa con un $75 \%$. Para ese mismo porcentaje, es evidente la relación entre la presencia de dilemas bioéticos y el incremento del síndrome de Burn-out en el personal médico.

Sobre la delegación de decisiones de los casos con dilema ético por el personal a cargo, el $87,5 \%$ senala que no lo realiza. La respuesta de los participantes a la cuestión de si existe temor a posibles demandas jurídicas fue afirmativa en el 93,7 \%.

En la pregunta sobre si la toma de alguna decisión en torno a dilemas tales como limitación del esfuerzo terapéutico (LET), orden de no reanimación (ONR) o negativa de ingreso a UTIP, genera duda o indecisión, se obtuvo respuesta afirmativa en $81,2 \%$ y negativa en $18,7 \%$.

La información obtenida con relación a la posible repercusión personal que conlleva tomar este tipo de decisiones, fue afirmativa en $56,2 \%$, y negativa en $25 \%$, quedando sin respuesta en $18,7 \%$. Se mencionó en forma descendente la presencia de ansiedad, inseguridad, depresión, inconformidad y preocupación.

En la segunda y última fase de la encuesta los participantes tuvieron acceso a la totalidad de las respuestas, bajo anonimato del nombre del médico y del centro de trabajo, con la intención de saber si deseaban hacer una modificación. La totalidad de los médicos ratificaron sus respuestas (tabla 1).

\section{Discusión}

Entre las virtudes humanas, la más difícil de ejercer es la prudencia, y precisamente la acción prudencial se requiere en el manejo de pacientes terminales y, evidentemente, en toda decisión difícil en torno a la atención de pacientes críticamente enfermos. La prudencia, al incidir sobre todas las demás virtudes, es una virtud intelectual y también moral, porque perfecciona al hombre en forma integral. Una propiedad típica de las virtudes es la de ser aptas para encontrar el medio adecuado entre dos extremos que ocasionan error. En el caso de pacientes terminales, estos dos extremos son la obstinación terapéutica y el abandono del paciente: en el primer caso se realizan acciones que no deberían realizarse; en el segundo, se dejan de ejecutar aquellas que sí deberían serlo.

El proceso de deliberación clínica y ética es el fundamento reflexivo que facilita la difícil toma de decisiones. Mediante este proceso se determinan los factores que intervienen en un acto o situación concreta, con el fin de buscar su solución óptima o, cuando esto no es posible, la menos lesiva(8). A través de la deliberación, se busca analizar los problemas en toda su complejidad, enfatizar los principios y valores implícitos, y las circunstancias, consecuencias y aspectos jurídicos.

Tabla 1: Resultados de encuesta

\begin{tabular}{|c|c|c|c|}
\hline PREGUNTA & Sí & No & Sin respuesta \\
\hline ¿La relación médico-paciente-familia se ha visto afectada? & $32,5 \%$ & $67,5 \%$ & \\
\hline ¿Ha enfrentado demandas? & $37,6 \%$ & $62,5 \%$ & \\
\hline ¿La relación interpersonal con el resto del equipo médico se ha visto afectada? & $25 \%$ & $75 \%$ & \\
\hline ¿Ha motivado incremento del síndrome de Burn out? & $75 \%$ & $25 \%$ & \\
\hline ¿Le motiva delegar en otro médico la responsabilidad de los casos con dilema ético? & $12,5 \%$ & $87,5 \%$ & \\
\hline ¿Existe temor a posibles demandas jurídicas? & $93,7 \%$ & $6,3 \%$ & \\
\hline $\begin{array}{l}\text { ¿Al tomar una decisión en torno a dilemas (limitación del esfuerzo terapéutico, } \\
\text { orden de no reanimación, negativa de ingreso a UTIP), existe duda si es éticamente } \\
\text { correcta? }\end{array}$ & $81,2 \%$ & $18,7 \%$ & \\
\hline $\begin{array}{l}\text { ¿Ha repercutido en forma personal el tomar este tipo de decisiones? (ansiedad, } \\
\text { depresión, molestia etc.) }\end{array}$ & $56,2 \%$ & $25 \%$ & $18,7 \%$ \\
\hline $\begin{array}{l}\text { Otro (s) } \\
\text { Cuál (es) }\end{array}$ & & & $100 \%$ \\
\hline
\end{tabular}


E. Pellegrino(9) ha insistido por varias décadas en la importancia de la enseñanza de la ética de las virtudes en la formación y el ejercicio de la medicina; consideramos que, en el caso que nos convoca, esta premisa se observa evidente. Es en las UTIP, ante el paciente que no puede comunicarse, ni tiene competencia, donde paradójicamente surgen los más complejos dilemas éticos, en torno al fin de la vida, a la donación de órganos, a la "muerte digna" en pacientes terminales, a la indicación de no reanimación y a la relevante relación médico-paciente-familia. Reconociendo que existe un continuo debate acerca de las consecuencias sociales, económicas, culturales, religiosas y morales que motivan establecer adecuaciones y límites en el otorgamiento de los cuidados inten$\operatorname{sivos}(10,11)$.

Diversos factores contribuyen al desbalance en el manejo del paciente. Consideramos que son dos los que más influyen en la práctica. Uno de ellos está relacionado con la formación profesional. Por generaciones se había insistido en que el médico debía conservar la vida de sus pacientes a toda costa, pero en la práctica esta recomendación se encontraba matizada por la misma realidad, ya que llegaba un momento en que los recursos terapéuticos y los esfuerzos médicos chocaban con el hecho contundente de la muerte. A fin de cuentas, la naturaleza o el destino seguía su curso y pocos eran los pacientes que continuaban con vida después de un paro cardio-respiratorio. Había un equilibrio natural entre lo que el médico podía hacer y sus resultados.

$\mathrm{Al}$ incorporarse a las unidades de terapia intensiva, el uso de la ventilación mecánica, el desfibrilador, así como otros apoyos medicamentosos, como los fármacos inotrópicos y vasopresores, el panorama cambió y entonces los esfuerzos de reanimación fueron más efectivos. En forma paralela, aparece el concepto de muerte cerebral y entidades nosológicas nuevas, como el estado vegetativo persistente y las agonías prolongadas. En consecuencia, un cambio se suscitó en la visión del ejercicio médico: para lograr mantener la vida a toda costa, era necesario usar tecnología a toda costa. Con esta fórmula, muchos médicos, con absoluta buena fe, consideraron que la obstinación terapéutica no era tal, sino que solamente se trataba de hacer lo mejor posible desde el mundo de la tecnología.
Pero en ese planteamiento existe un error, porque dado que la condición humana es inseparable de la enfermedad, el sufrimiento y la muerte, la medicina debería aceptar la finitud humana y enseñar o ayudar a vivir en ella. Por el beneficio del paciente, la medicina debe procurar ser fiel a sí misma y mantenerse como algo viable y sostenible, y no dejarse arrastrar por un desarrollo tecnológico imparable.

Otro grave problema, que genera actitudes polarizadas en el manejo de pacientes terminales, es el de las actitudes médicas marcadas por mecanismos de defensa, que, aunque inconscientes, son reales en el momento de tomar decisiones. Los médicos, como cualquier ser humano, presentan conflictos existenciales que, ante situaciones límite, pueden hacer evidente su falta de resolución. La muerte y el sufrimiento provocan sentimientos encontrados y el médico, al no haber resuelto su propio conflicto, y tratando de sobreponerse a su miedo, reacciona, en vez de razonar, con actitudes de superioridad, como en el caso de la obstinación, o de evasión y huida, como en el de abandono del paciente. La actitud es evidente en el paso de la visita médica y el dictado de las indicaciones. Entre las frases "se hará todo lo que se pueda..." y el "no tiene caso hacer nada..." se encuentran encubiertos temores ocultos que buscan liberar angustia.

Por un extremo, hay personas a las que el miedo les hace refugiarse en la tecnología, que como Dios griego siempre es poderoso y da sensación de seguridad. Una de las alternativas de reacción ante el miedo es el ataque, por eso se pretende utilizar toda la tecnología al alcance, ya que la muerte del paciente representaría un fracaso personal, así como el enfrentamiento con la posibilidad de la muerte propia, a la cual se teme. "Nadie debe morir, pues si existe la muerte, yo moriré también", podría ser el pensamiento inconsciente que anima esta actitud.

Otra alternativa psicológica es la huida, pretender que ese paciente no existe ya bajo nuestro cuidado, en evitar su presencia. Se trata de un mecanismo de evasión. También esta actitud habla de un conflicto no resuelto ante la muerte. "No sé confortar, ni ayudar emocionalmente, el sufrimiento del paciente es demasiado para mi, me hace 
sufrir, y yo no quiero sufrir", nuevamente es el pensamiento inconsciente que alienta esta forma de conducta(12).

Si bien ambas acciones se comprenden, no se justifican en la acción profesional. En este sentido, el personal responsable deberá afrontar sus temores $y$, por el bien del paciente y el suyo, encontrar soluciones con sentido racional y humano para el buen hacer de su profesión.

En gran parte esta contribución puede ser dada por la visión científica y ética de los cuidados paliativos. Este tipo de manejo permite, por una parte, reconocer realidades $y$, por otra, ofrecer alternativas. No existe nada más desgastante, psicológicamente hablando, que la sensación de indefensión. El médico necesita saber que sí está haciendo un acto médico valioso y sumamente útil para su paciente, que es parte de su profesión y su vocación, y que, a la vez, le ayudará a encontrar firmeza en sus decisiones ante la realidad humana.

Verdaderamente, sí se puede "hacer todo lo que se pueda hacer", pero sin perder la objetividad. Cada caso tiene particularidades propias, exige el mayor esfuerzo y requiere que el personal de salud afronte la muerte en su ejercicio profesional(13).

En términos metodológicos, en el presente estudio se aprecia una adecuada participación, dado que hubo respuesta de médicos con experiencia que laboran en seis de las siete UTIP invitadas a participar, considerándose como una muestra significativa el $85 \%$ obtenido. La evidencia recopilada muestra la necesidad subyacente en el personal médico de las UTIP de implementar la reflexión ética en forma oportuna dentro de las guías de diagnóstico y tratamiento. Existe el deseo de contar con mayor conocimiento en el campo de la bioética, para enfrentar el trabajo cotidiano y fortalecer acuerdos internos sobre la base de debate o consenso. Lo anterior se hizo evidente al existir discrepancias en los conceptos expresados por algunos médicos que laboran en la misma UTIP, considerando que hace falta uniformar conceptos sobre aspectos éticos que optimicen las decisiones, que respeten los intereses de todos los involucrados (paciente, familia, personal de salud y de la propia institución), en suma, que respondan a la necesidad de unir el conocimiento científico de la medicina basada en evidencias, con el sentido humano y de prevención de conflictos que representa la medicina basada en reflexión.

El interés que manifestó el personal médico en la enseñanza de la bioética posiblemente obedece al hecho de que, en la actualidad, se incluye solo un curso básico, no presencial ni llevado a la práctica diaria, sobre este importante aspecto que sustenta la buena práctica médica.

Algunos autores hacen referencia a la importancia de la participación activa de los padres o tutores en la toma de decisiones(14), hecho contrario a lo referido como posible solución en la presente encuesta. Buscando el mejor interés del niño, a diferencia del adulto, es conveniente, en ocasiones, dejar la autonomía en segunda consideración y apoyarse en un paternalismo "benigno" que colabore en la decisión final $(15,16)$. Deberá valorarse, bajo situaciones inesperadas y críticas que rebasan la comprensión de la familia y no brindan tiempo a la reflexión, si resulta inadecuado apoyarse totalmente en la autonomía de la familia o del tutor responsable, que además, en consecuencia, tiene alterado su estado anímico. Finalmente, la autonomía, reflejada en el consentimiento informado, debe ser considerada como un proceso de información que asegure la comprensión y alcance de las decisiones, y no solo el momento de firmar un documento(17).

En el contexto de soluciones posibles a los dilemas bioéticos, se menciona la creación de unidades de cuidados paliativos, las cuales, favorablemente, han tenido en nuestro país una progresiva expansión en los hospitales.

Es evidente que los comités hospitalarios de bioética, aunque presentes en las unidades hospitalarias, no brindan completo apoyo en casos problema de las unidades de cuidados intensivos. Ello probablemente se deba a que, en forma idónea, aunque no fácil, debe contar con un comité de expertos las 24 horas, adecuándose a la dinámica de las UTIP, y coadyuvar a la comprensión del difícil proceso en la toma de decisiones, tanto a la familia como al médico, en un intento de minimizar el desgaste físico-emocional cotidiano. La actual tecnología de fácil presencia virtual podría facilitar la intercomunicación. 
En el ámbito de las decisiones, a veces difíciles, en la atención del niño grave, es importante apoyarse en una adecuada conceptualización de términos inmersos en el lenguaje del paciente crítico y de la bioética. Evitemos una común confusión entre el significado de "eutanasia" y su clasificación en pasiva o activa, acción no procedente en la atención de niños críticamente enfermos, que además suele confundirse con "limitación o adecuación del esfuerzo terapéutico"(17), acción necesaria solo en pacientes previamente diagnosticados como terminales, en quienes deja de existir alguna posibilidad terapéutica eficaz, debiendo ofrecer cuidados paliativos que favorezcan su bienestar, evitando con ello la obstinación terapéutica. Sin embargo, en algunas ocasiones se considera erróneamente la no instauración de medidas terapéuticas como eutanasia pasiva(16-19).

Es evidente la preocupación del personal médico ante la posibilidad de enfrentar demandas jurídicas, reconociendo que una tercera parte de ellos la ha experimentado. Gran parte de los conflictos se relaciona con aspectos de comunicación, con el enfoque ético de la relación médico-familia y con valores sustentados. Encontrar posibles abordajes favorecerá una buena relación médico/paciente/ equipo de salud y una disminución de las quejas y demandas en estos servicios(20).

Acorde con la respuesta negativa -en un $87,5 \%$ - sobre delegar en otro médico la responsabilidad de los casos con dilema ético, se reconoce el interés de los médicos por acompañar siempre al paciente y a su familia durante el proceso de atención, favoreciendo una adecuada relación. Durante la formación profesional, al médico se le transmite que la medicina "científica" es casi infa- lible. Pero la realidad es otra. El hecho es que, por la gran cantidad de variables que se presentan en un acto médico, este nunca puede ser determinado, sino probabilístico; aun aplicando la ciencia más exacta a un caso específico no hay garantía sino probabilidad de éxito. Sucede que siempre existen situaciones de incertidumbre, en las que la forma racional de tomar decisiones termina siendo la prudencia $(21,22)$. Por eso, a fin de cuentas, el quehacer médico se convierte no en ciencia exacta, sino en ciencia prudencial, y con ello volvemos a señalar la importancia del desarrollo de esta virtud.

La práctica de la medicina en la UTIP se complica en el campo ético precisamente por la indefensión y falta de autonomía de nuestros pacientes. Los médicos debemos partir entonces y siempre del principio de beneficencia, mediante una sobresaliente relación con los padres del enfermo, para evitar incorrectas interpretaciones sobre el manejo elegido y, ante todo, mediante una formación recta de conciencia, para distinguir siempre lo correcto de lo incorrecto y así tener mayor certeza en las decisiones tomadas.

Incluir en forma cotidiana la evaluación bioética y la toma de decisiones con contenido moral en la formación de estos profesionistas, convencidos de que cada caso es diferente en su entorno familiar y de valores, así como también fortalecer la indispensable relación, sustentada en comunicación asertiva, entre el equipo de salud y la familia, y promover el funcionamiento adecuado de comités de bioética hospitalarios con funciones consultivas de emergencia, son propuestas de los autores. 
Referencias

1. García RH, Limón Ll. Bioética general. Trillas; 2009.

2. Beauchamp LT, Childress JF. Principles of Biomedical Ethics. Nueva York: Oxford University Press; 1989.

3. Taboada P, López R. Metodología de análisis ético de casos clínicos. En García JJ, (dir.) Enciclopedia de Bioética. Disponible en: http://enciclopediadebioetica.com/index.php/todas-las-voces/153. Consultado el 14/11/13.

4. Hernández Rastrollo R, Hernández González A, Hna. Tezanos MT, et al. Grupo de Ética de la Sociedad Española de Cuidados Intensivos Pediátricos. An Pediatr (Barc) 2008; 68(4): 393-400.

5. Pollack MM, Ruttiman UE, Getson PR. Pediatric risk of mortality (PRIMS) score. Crit Care Med 1988; 16: 11101116.

6. Fuhrman BP, Zimmerman JJ. Pediatric Critical Care. Mosby Year Book; 2006.

7. Casanueva Mateos L, Ruiz López P, Sánchez Díaz JI, et al. Cuidados al final de la vida en la unidad de cuidados intensivos pediátrica. Revisión de la bibliografía. An Pediatr (Barc) 2005; 63(2): 152-159.

8. Gracia D. La deliberación moral: el método de la ética clínica. Med Clin (Barc) 2001; 117: 18-23.

9. Pellegrino ED. The virtues in medical practice. Oxford University Press US; 1993.

10. Keenan HT, Diekema DS, O'Rourke PP, Cummings P, Woodrum DE. Attitudes toward limitation of support in a pediatric intensive care unit. Crit Care Med 2000 May; 28(5): 1590.

11. De Araújo Torreáo L, Romeu Pereira C, Troster E. Ethical aspects in the management of the terminally ill patient in the Pediatric Intensive Unit. Rev Hosp Clin Fac Med S Paulo 2004; 59(1): 3-9.

12. Casas ML. Conferencia magistral. Simposio sobre Aspectos Éticos de la Eutanasia, Comisión Nacional Mexicana de Bioética/SSA. 23 enero 2006. Aceptado para publicación por SSA/CNMB.

13. Harris CC, McNicholas JJ. Paediatric intensive care in the field hospital. J R Army Med Corps 2009 Jun; 155 (2): $157-$ 159.

14. Shields L, Pratt J, Davis L, Hunter J. Family-centred care for children in hospital. Disponible en http://www.ncbi.nlm. nih.gov/pubmed/17253525. Consultado el 14/11/13.

15. Bustamante MA. Limitación terapéutica y conflictos éticos. Proporcionalidad. Revista Argentina de Medicina Respiratoria 2006; 6(3).

16. Ratnapalan M, Cooper AB, Scales DC, Pinto R. Documentation of best interest by intensivists: a retrospective study in an Ontario critical care unit. BMC Medical Ethics 2010; 11:1. Disponible en: http://www.biomedcentral.com/14726939/11/1 Consultado el 14/11/13.

17. Frader JE. Forgoing life support across borders: who decides and why. Pediatr Crit Care Med 2004; 5: 289-290.

18. Tibballs J. Legal basis for ethical withholding and withdrawing life-sustaining medical treatment from infants and children. J Paediatr Child Health 2007 Apr; 43(4): 230-236.

19. Billings JA. Humane terminal extubation reconsidered: The role for preemptive analgesia and sedation. Crit Care Med 2012; 40(2): 625-630.

20. Giannini A, Messeri A, Aprile A, Casalone C, Jankovic M. Recommendations of the Italian Society of Neonatal and Pediatric Anesthesia and Intensive Care (SARNePI). End-of-life decisions in pediatric intensive care. Pediatric Anesthesia 2008; 18: 1089-1095.

21. Oliveros Donohue M. Ethics and poor prognostic conditions. Rev Peru Pediatr 2008; 61(2).

22. Lago PM, Devictor D, Piva JP, Bergounioux J. Care in children: the Brazilian and the international perspectives. Jornal de Pediatria 2007; 83(2) (Suppl).

Recibido: 12 de mayo de 2012

Aceptado: 14 de agosto de 2012 